DOI: $10.2478 / \mathrm{v} 10324-012-0010-7$<smiles>C1=C2C=C(C1)C2</smiles>

VERSITA
Analele Universităţii de Vest,

Timişoara

Seria Matematică - Informatică

L, 1, (2012), 115- 125

\title{
Notes on Continuous Parameter Periodically $\Gamma$-correlated Processes
}

Ilie Valuşescu

\begin{abstract}
A $\Gamma$-correlated process is a stochastic process considered in the context of a complete correlated action $\{\mathcal{E}, \mathcal{H}, \Gamma\}$. In this note continuous parameter periodically $\Gamma$-correlated processes are analyzed, a Gladyshev's type theorem is extended to the $\{\mathcal{E}, \mathcal{H}, \Gamma\}$ case, and some consideration on continuous time parameter harmonizable $\Gamma$-correlated processes are made.
\end{abstract}

AMS Subject Classification (2000). 47N30, 47A20, 60G25

Keywords. Complete correlated actions, continuous time periodically $\Gamma$-correlated processes, semispectral measures, harmonizability.

\section{Preliminaries}

Let $\mathcal{L}(\mathcal{E})$ be the $C^{*}$-algebra of all linear bounded operators on a separable Hilbert space $\mathcal{E}$, and $\mathcal{H}$ a right $\mathcal{L}(\mathcal{E})$-module. An action of $\mathcal{L}(\mathcal{E})$ on $\mathcal{H}$ is given considering $A h:=h A$ in the sense of the right $\mathcal{L}(\mathcal{E})$-module. The action of $\mathcal{L}(\mathcal{E})$ on $\mathcal{H}$ is correlated if there exists a function (the correlation of the action) $\Gamma: \mathcal{H} \times \mathcal{H}$ into $\mathcal{L}(\mathcal{E})$ given by $(h, g) \rightarrow \Gamma[h, g]$, such that

(i) $\Gamma[h, h] \geq 0, \Gamma[h, h]=0 \quad$ implies $h=0$;

(ii) $\Gamma[g, h]=\Gamma[h, g]^{*}$;

(iii) $\Gamma[h, A g]=\Gamma[h, g] A$

In various proofs the following formula, inferred by (ii) and (iii), is used. 


$$
\Gamma\left[\sum_{i} A_{i} h_{i}, \sum_{j} B_{j} g_{j}\right]=\sum_{i, j} A_{i}^{*} \Gamma\left[h_{i}, g_{j}\right] B_{j},
$$

for finite sums of actions of $\mathcal{L}(\mathcal{E})$ on $\mathcal{H}$.

A triple $\{\mathcal{E}, \mathcal{H}, \Gamma\}$ defined as above was called [7] a correlated action of $\mathcal{L}(\mathcal{E})$ on $\mathcal{H}$.

An example of correlated action can be constructed as follows. Take $\mathcal{H}=$ $\mathcal{L}(\mathcal{E}, \mathcal{K})$ - the space of the linear bounded operators from $\mathcal{E}$ into $\mathcal{K}$, where $\mathcal{E}$ and $\mathcal{K}$ are Hilbert spaces. An action of $\mathcal{L}(\mathcal{E})$ on $\mathcal{L}(\mathcal{E}, \mathcal{K})$ is given if we consider $A V:=V A$ for each $A \in \mathcal{L}(\mathcal{E})$ and $V \in \mathcal{L}(\mathcal{E}, \mathcal{K})$. It is easy to see that $\Gamma\left[V_{1}, V_{2}\right]=V_{1}^{*} V_{2}$ is a correlation of the action of $\mathcal{L}(\mathcal{E})$ on $\mathcal{L}(\mathcal{E}, \mathcal{K})$, and the triple $\{\mathcal{E}, \mathcal{L}(\mathcal{E}, \mathcal{K}), \Gamma\}$ is a correlated action. In [7] was proved that for any correlated action $\{\mathcal{E}, \mathcal{H}, \Gamma\}$ there exists a Hilbert space $\mathcal{K}$ and an algebraic imbedding $h \rightarrow V_{h}$ of $\mathcal{H}$ into $\mathcal{L}(\mathcal{E}, \mathcal{K})$.

Such a way, if we consider as the state space to be a right $\mathcal{L}(\mathcal{E})$-module $\mathcal{H}$, the behavior of a process $\left\{f_{t}\right\}_{t \in G}$ from $\mathcal{H}$ can be studied knowing the behavior of the operatorial process $\left\{V_{f_{t}}\right\}$ from $\mathcal{L}(\mathcal{E}, \mathcal{K})$. The corresponding space $\mathcal{K}$ is called the measuring space and $\mathcal{E}$ is the parameter space. If the algebraic imbedding of $\mathcal{H}$ into $\mathcal{L}(\mathcal{E}, \mathcal{K})$ is onto, then the correlated action $\{\mathcal{E}, \mathcal{H}, \Gamma\}$ is a complete correlated action.

Since in $\mathcal{H}$ we have not a proper orthogonal projection on a right $\mathcal{L}(\mathcal{E})$ submodule, a $\Gamma$-orthogonal projection was constructed, using the following Proposition, helping to solve specific prediction problems.

Proposition 1.1. Let $\mathcal{H}_{1}$ be a submodule in the right $\mathcal{L}(\mathcal{E})$-module $\mathcal{H}$ and

$$
\mathcal{K}_{1}=\bigvee_{x \in \mathcal{H}_{1}} V_{x} \mathcal{E} \subset \mathcal{K}
$$

For each $h \in \mathcal{H}$ there exists a unique element $h_{1} \in \mathcal{H}$ such that for each $a \in \mathcal{E}$ we have

$$
V_{h_{1}} a \in \mathcal{K}_{1} \quad \text { and } \quad V_{h-h_{1}} a \in \mathcal{K}_{1}^{\perp} .
$$

Moreover, we have

$$
\Gamma\left[h-h_{1}, h-h_{1}\right]=\inf _{x \in \mathcal{H}_{1}} \Gamma[h-x, h-x],
$$

where the infimum is taken in the set of all positive operators from $\mathcal{L}(\mathcal{E})$.

For a complete proof see e.g. [8] or [9].

To a process $\left\{f_{t}\right\}_{t \in G}$ from $\mathcal{H}$, where $G$ is $\mathbb{Z}, \mathbb{R}$, a locally compact group or a hypergroup, a correlation function is attached by

$$
\Gamma_{f}(s, t)=\Gamma\left[f_{s}, f_{t}\right] .
$$


Also, for two processes $\left\{f_{t}\right\}_{t \in G}$ and $\left\{g_{t}\right\}_{t \in G}$ a cross-correlation function is attached by

$$
\Gamma_{f g}(s, t)=\Gamma\left[f_{s}, g_{t}\right]
$$

If the correlation function depends only on the difference $t-s$, not on $s$ and $t$ separately, then the process $\left\{f_{t}\right\}_{t \in G}$ is called $\Gamma$-stationary, otherwise the process is a nonstationary one. Similarly, if the cross-correlation function $\Gamma_{f g}(s, t)$ depends only on the difference $t-s$, then $\left\{f_{t}\right\}_{t \in G}$ and $\left\{g_{t}\right\}_{t \in G}$ are stationary cross-correlated processes. Of course, in the $\Gamma$-stationary case (1.4) becomes

$$
\Gamma_{f}(t)=\Gamma\left[f_{0}, f_{t}\right]
$$

If $G=\mathbb{Z}$, then $\left\{f_{n}\right\}_{n \in \mathbb{Z}} \in \mathcal{H}$ is a discrete $\Gamma$-stationary process and an exhaustive prediction study can be found e.g. in [8], [9]. In the continous case $(G=\mathbb{R})$ some investigations for $\Gamma$-correlated processes was done in [10].

The main investigation tools was the using of $L^{2}$-bounded analytic functions instead of bounded analytic functions, and a study of $\mathcal{L}(\mathcal{E})$-valued semispectral measures. An $\mathcal{L}(\mathcal{E})$-valued semispectral measure is a map $\sigma \rightarrow F(\sigma)$ from the family $\mathcal{B}(\mathbb{T})$ of Borel subsets of the unit torus $\mathbb{T}$ from the complex plane $\mathbb{C}$ into $\mathcal{L}(\mathcal{E})$ such that for any $a \in \mathcal{E}$ the map $\sigma \rightarrow\langle F(\sigma) a, a\rangle$ is a positive Radon measure on $\mathbb{T}$. An $L^{2}$-bounded operator valued analytic function is an analytic function $\Theta(\lambda)=\sum_{n=0}^{\infty} \Theta_{n} \lambda^{n}$ on the open unit disc $\mathbb{D}$, where the operator coefficients $\Theta_{n} \in \mathcal{L}(\mathcal{E}, \mathcal{F})$, such that there exists $M>0$ verifying

$$
\sum_{n=0}^{\infty}\left\|\Theta_{n} a\right\|^{2} \leq M^{2}\|a\|^{2} \quad(a \in \mathcal{E})
$$

or equivalently,

$$
\sup _{0 \leq r<1} \frac{1}{2 \pi} \int_{0}^{2 \pi}\left\|\Theta\left(r \mathrm{e}^{i t}\right) a\right\|^{2} \mathrm{~d} t \leq M^{2}\|a\|^{2} \quad(a \in \mathcal{E}) .
$$

An operator valued analytic function is denoted usually by a triplet $\{\mathcal{E}, \mathcal{F}, \Theta(\lambda)\}$. Also a central role in the study of infinite variate $\Gamma$-stationary processes played the generalized Lowdenslager-Sz.-Nagy-Foiaş factorization theorem, which attach to each semispectral measure $F$ a maximal outer $L^{2}$-bounded function, so called the maximal function of $F$. 


\section{Continuity conditions}

In this note we are mainly interested in the study of processes $\left\{f_{t}\right\}_{t \in \mathbb{R}}$ in the context of a complete correlated action $\{\mathcal{E}, \mathcal{H}, \Gamma\}$.

For a process $\left\{f_{t}\right\}_{t \in \mathbb{R}}$ from $\mathcal{H}$ in the complete correlated action $\{\mathcal{E}, \mathcal{H}, \Gamma\}$, the space generated by $\left\{f_{t}\right\}_{t \in \mathbb{R}}$ in the right $\mathcal{L}(\mathcal{E})$-module $\mathcal{H}$ is defined by

$$
\mathcal{H}_{\infty}^{f}=\left\{A_{t} f_{t} ; t \in \mathbb{R}\right\},
$$

as a right $\mathcal{L}(\mathcal{E})$-submodule of $\mathcal{H}$, and by the algebraic imbedding $h \rightarrow V_{h}$ of $\mathcal{H}$ into $\mathcal{L}(\mathcal{E}, \mathcal{K})$, to $\mathcal{H}_{\infty}^{f}$ in the measuring space $\mathcal{K}$ corresponds the following subspace

$$
\mathcal{K}_{\infty}^{f}=\bigvee_{t \in \mathbb{R}} V_{f_{t}} \mathcal{E}=\bigvee_{g \in \mathcal{H}_{\infty}^{f}} V_{g} \mathcal{E} .
$$

A process $\left\{f_{t}\right\}_{t \in \mathbb{R}}$ is said to be norm, strongly, or weakly continuous, if $t \rightarrow V_{f_{t}}$ is norm, strongly, or weakly continuous in $\mathcal{L}(\mathcal{E}, \mathcal{K})$. The process $\left\{f_{t}\right\}_{t \in \mathbb{R}}$ is bounded if there is a positive constant $M>0$ such that $\left\|V_{f_{t}}\right\| \leq M$ for $t \in \mathbb{R}$. As we have seen in the previous part, to a process $\left\{f_{t}\right\}_{t \in \mathbb{R}}$ an operatorial valued correlation function $\Gamma(s, t)$ is attached by (1.4), and for any $a, b \in \mathcal{E}$ a scalar correlation function $\tilde{\Gamma}_{a b}(s, t): \mathbb{R} \times \mathbb{R} \rightarrow \mathbb{C}$ is defined by

$$
\tilde{\Gamma}_{a b}(s, t)=\langle\Gamma(s, t) a, b\rangle_{\varepsilon} .
$$

Proposition 2.1. The process $\left\{f_{t}\right\}_{t \in \mathbb{R}} \in \mathcal{H}$ is strongly continuous if and only if for any $a, b \in \mathcal{E}$ the scalar correlation function $\tilde{\Gamma}_{a b}(s, t)$ is continuous.

Proof. We have only to see that

$$
\begin{gathered}
\left|\tilde{\Gamma}_{a b}(s+u, t+v)-\tilde{\Gamma}_{a b}(s, t)\right|=\left|\left\langle\Gamma\left[f_{s+u}, f_{t+v}\right] a, b\right\rangle_{\varepsilon}-\left\langle\Gamma\left[f_{s}, f_{t}\right] a, b\right\rangle_{\varepsilon}\right| \leq \\
\leq\left|\left\langle\Gamma\left[f_{s+u}-f_{s}, f_{t+v}\right] a, b\right\rangle_{\varepsilon}\right|+\left|\left\langle\Gamma\left[f_{s}, f_{t+v}-f_{t}\right] a, b\right\rangle_{\varepsilon}\right| \leq \\
\leq\left[\left\|\Gamma\left[f_{s+t}-f_{s}, f_{t+v}\right] a\right\|+\left\|\Gamma\left[f_{s}, f_{t+v}-f_{t}\right] a\right\|\right]\|b\|= \\
=\left[\left\|V_{f_{s+t}-f_{s}}^{*} V_{f_{t+v}} a\right\|+\left\|V_{f_{s}}^{*} V_{f_{t+v}-f_{t}} a\right\|\right]\|b\| \leq \\
\leq\left[\left\|\left(V_{f_{s+u}}-V_{f_{s}}\right)^{*} V_{f_{t+v}} a\right\|+\left\|V_{f_{s}}\right\|\left\|V_{f_{t+v}} a-V_{f_{t}} a\right\|\right]\|b\| \rightarrow 0
\end{gathered}
$$

as $u, v \rightarrow 0$.

Conversely, if $\tilde{\Gamma}_{a b}(s, t)$ is a continuous function on $\mathbb{R} \times \mathbb{R}$ for any $a, b \in \mathcal{E}$, then for any $u \in \mathbb{R}$ we have

$$
\left\|V_{f_{t+u}} a-V_{f_{t}} a\right\|_{\mathcal{K}}^{2}=\left\langle V_{f_{t+u}} a-V_{f_{t}} a, V_{f_{t+u}} a-V_{f_{t}} a\right\rangle_{\mathcal{K}}=
$$




$$
\begin{gathered}
=\left\langle V_{f_{t+u}} a, V_{f_{t+u}} a\right\rangle-\left\langle V_{f_{t+u}} a, V_{f_{t}} a\right\rangle-\left\langle V_{V_{f_{t}}} a, V_{f_{t+u}} a\right\rangle+\left\langle V_{f_{t}} a, V_{f_{t}} a\right\rangle= \\
=\left\langle V_{f_{t+u}}^{*} V_{f_{t+u}} a, a\right\rangle-\left\langle V_{f_{t}}^{*} V_{f_{t+u}} a, a\right\rangle-\left\langle V_{f_{t+u}}^{*} V_{f_{t}} a, a\right\rangle+\left\langle V_{f_{t}}^{*} V_{f_{t}} a, a\right\rangle= \\
=\langle\Gamma(t+u, t+u) a, a\rangle-\langle\Gamma(t, t+u) a, a\rangle-\langle\Gamma(t+u, t) a, a\rangle+\langle\Gamma(t, t) a, a\rangle= \\
=\tilde{\Gamma}_{a a}(t+u, t+u)-\tilde{\Gamma}_{a a}(t, t+u)-\tilde{\Gamma}_{a a}(t+u, t)+\tilde{\Gamma}_{a a}(t, t) \rightarrow 0
\end{gathered}
$$

as $u$ converges to zero.

Proposition 2.2. If $\left\{f_{t}\right\}_{t \in \mathbb{R}}$ is a weakly continuous process, then for any $a, b \in \mathcal{E}$ the scalar correlation function $\tilde{\Gamma}_{a b}(s, t)$ is separately continuous on $\mathbb{R} \times \mathbb{R}$.

Conversely, if $\left\{f_{t}\right\}_{t \in \mathbb{R}}$ is bounded and $\tilde{\Gamma}_{a b}(s, t)$ is separately continuous on $\mathbb{R} \times \mathbb{R}$, then $\left\{f_{t}\right\}_{t \in \mathbb{R}}$ is weakly continuous.

Proof. Obviously we have

$$
\begin{gathered}
\left|\tilde{\Gamma}_{a b}(s+u, t)-\tilde{\Gamma}_{a b}(s, t)\right|=\left|\langle\Gamma(s+u, t) a, b\rangle_{\varepsilon}-\langle\Gamma(s, t) a, b\rangle_{\varepsilon}\right|= \\
=\left\langle V_{f_{s+u}}^{*} V_{f_{t}} a, b\right\rangle_{\varepsilon}-\left\langle V_{f_{s}}^{*} V_{f_{t}} a, b\right\rangle_{\varepsilon}=\left\langle\left(V_{f_{s+u}}-V_{f_{s}}\right)^{*} V_{f_{t}} a, b\right\rangle_{\varepsilon}= \\
=\left\langle V_{f_{t}} a,\left(V_{f_{s+u}}-V_{f_{s}}\right) b\right\rangle_{\mathcal{K}} \rightarrow 0
\end{gathered}
$$

as $u \rightarrow 0$.

Analogously we have that for any $a, b \in \mathcal{E}$ and $v \in \mathbb{R}$

$$
\left|\tilde{\Gamma}_{a b}(s, t+v)-\tilde{\Gamma}_{a b}(s, t)\right| \rightarrow 0
$$

as $v \rightarrow 0$.

If $\left\{f_{t}\right\}_{t \in \mathbb{R}}$ is bounded and for any $a, b \in \mathcal{E}$ the scalar correlation function $\tilde{\Gamma}_{a b}(s, t)$ is separately continuous, then for $s \in \mathbb{R}$ the function $\tilde{\Gamma}_{a b}(\cdot, s)$ is continuous, and for any $y=\sum_{j=1}^{n} \overline{\alpha_{j}} V_{f_{s_{j}}} b$ from $\mathcal{K}_{\infty}^{f}$ the function

$$
\left\langle V_{f .} a, y\right\rangle_{\mathcal{K}}=\sum_{j=1}^{n} \alpha_{j}\left\langle V_{f .} a, V_{f_{s_{j}}} b\right\rangle=\sum_{j=1}^{n} \alpha_{j} \tilde{\Gamma}_{a b}\left(\cdot, s_{j}\right)
$$

is continuous. Choosing in $\mathcal{K}_{\infty}^{f}$ a sequence $\left\{k_{n}\right\}_{n=1}^{\infty}$, where $k_{n}=\sum_{j=1}^{n} \alpha_{n, j} V_{f_{s_{n, j}}} b$, such that $\left\|k_{n}-y\right\|_{\mathcal{K}} \rightarrow 0$, and taking account that $\tilde{\Gamma}_{a b}(\cdot, t)$ is continuous, then $\left\langle V_{f .} a, y\right\rangle_{\mathcal{K}}=\lim _{n \rightarrow \infty}\left\langle V_{f .} a, k_{n}\right\rangle_{\mathcal{K}}$, which is a uniform limit since $\left\{f_{t}\right\}$ is bounded. Therefore $\left\{f_{t}\right\}$ is weakly continuous. 


\section{Periodically correlated processes}

Let $\left\{f_{t}\right\}_{t \in \mathbb{R}}$ be a periodically $\Gamma$-correlated process, i.e. a process in the complete correlated action $\{\mathcal{E}, \mathcal{H}, \Gamma\}$, whose correlation function $\Gamma_{f}(s, t)$ given by (1.4) satisfies the periodicity condition

$$
\Gamma_{f}(s+T, t+T)=\Gamma_{f}(s, t) \quad(s, t \in \mathbb{R})
$$

for a positive real number $T$. The smallest $T>0$ satisfying (3.1) is the period of the process $\left\{f_{t}\right\}_{t \in \mathbb{R}}$.

Analogously with the scalar case [1] the notion of an almost-periodically $\Gamma$ correlated process $\left\{f_{t}\right\}_{t \in \mathbb{R}}$ can be introduced under the condition that its correlation function $\Gamma_{f}(s, t)$ is uniformly continuous, and is an almost-periodic function with respect to $T$ in the sense of Bohr, but in this note we are concerned mainly on the periodic case.

As in the discrete case, to the continuous parameter process $\left\{f_{t}\right\}_{t \in \mathbb{R}}$, beside the correlation function $\Gamma_{f}(s, t)$, for any $t \in \mathbb{R}$ the covariance function is defined by

$$
B(s, t)=\Gamma_{f}(s+t, s) \quad(s \in \mathbb{R}),
$$

and of course we have conversely

$$
\Gamma_{f}(s, t)=B(t, s-t) \quad(s, \in \mathbb{R}) .
$$

Since $B(s, t)$ is an operator valued periodic function in $s$ with the same period $T$ as the process $\left\{f_{t}\right\}_{t \in \mathbb{R}}$, there exists the following Fourier representation

$$
B(s, t)=\sum_{k \in \mathbb{Z}} B_{k}(t) \exp \left(\frac{2 \pi i k s}{T}\right),
$$

where the $\mathcal{L}(\mathcal{E})$-valued coefficients $B_{k}(t)$ are given by

$$
B_{k}(t)=\frac{1}{T} \int_{0}^{T} B(s, t) \exp \left(\frac{-2 \pi i k s}{T}\right) \mathrm{d} s \quad(t \in \mathbb{R}, k \in \mathbb{Z}) .
$$

Following the Gladyshev's results [1] the following extension to the complete correlated case $\{\mathcal{E}, \mathcal{H}, \Gamma\}$ can be obtained.

Theorem 3.1. A norm continuous operator valued function $B(s, t)$ which satisfies condition (3.1) for every $t, s \in \mathbb{R}$ is the covariance function of some continuous periodically $\Gamma$-correlated process with the same period $T>0$ if and only if the $\mathcal{L}(\mathcal{E})$-valued functions $B_{j k},(j, k \in \mathbb{Z})$, satisfy the following positive definiteness property

$$
\sum_{p, q=1}^{n}\left\langle A_{p}^{*} B_{k_{p} k_{q}}\left(t_{p}-t_{q}\right) A_{q} a, a\right\rangle_{\mathcal{E}} \geq 0 \quad(a \in \mathcal{E}),
$$


for any $n \geq 1, k_{1}, \ldots, k_{n} \in \mathbb{Z}, t_{1}, \ldots, t_{n} \in \mathbb{R}$, and $A_{1}, \ldots, A_{n} \in \mathcal{L}(\mathcal{E})$, where

$$
B_{j k}(t)=B_{k-j}(t) \exp \left(\frac{2 \pi i j t}{T}\right) .
$$

Proof. Let us remark that the correlation function and the covariance function of an arbitrary $\Gamma$-correlated process $\left\{f_{t}\right\}_{t \in \mathbb{R}}$ are positive definite functions. Indeed, for any $n \geq 1, a_{1}, \ldots, a_{n} \in \mathcal{E}, t_{1}, \ldots, t_{n} \in \mathbb{R}$, and $A_{1}, \ldots, A_{n} \in$ $\mathcal{L}(\mathcal{E})$ we have, taking account that for a finite system of elements $\left\{a_{k}\right\} \subset \mathcal{E}$ there exists a system of operators $S_{k} \in \mathcal{L}(\mathcal{E})$ such that $a_{k}=S_{k} a$,

$$
\begin{gathered}
\sum_{p, q=1}^{n}\left\langle A_{p}^{*} \Gamma\left(t_{p}, t_{q}\right) A_{q} a_{q}, a_{p}\right\rangle_{\varepsilon}=\sum_{p, q=1}^{n}\left\langle A_{p}^{*} \Gamma\left[f_{t_{p}}, f_{t_{q}}\right] A_{q} a_{q}, a_{p}\right\rangle_{\varepsilon}= \\
=\sum_{p, q=1}^{n}\left\langle\Gamma\left[A_{p} f_{t_{p}}, A_{q} f_{t_{q}}\right] a_{q}, a_{p}\right\rangle_{\varepsilon}=\sum_{p, q=1}^{n}\left\langle\Gamma\left[A_{p} f_{t_{p}}, A_{q} f_{t_{q}}\right] S_{q} a, S_{p} a\right\rangle_{\varepsilon}= \\
=\left\langle\Gamma\left[\sum_{p=1}^{n} S_{p} A_{p} f_{t_{p}}, \sum_{q=1}^{n} S_{q} A_{q} f_{t_{q}}\right] a, a\right\rangle_{\varepsilon}=\langle\Gamma[h, h] a, a\rangle_{\varepsilon}= \\
=\left\langle V_{h}^{*} V_{h} a, a\right\rangle_{\mathcal{E}}=\left\|V_{h} a\right\|_{\mathcal{K}}^{2} \geq 0 .
\end{gathered}
$$

Taking into account (3.2) it follows that (3.6) is verified.

Conversely, if (3.6) is verified, then if we put

$$
B_{n}(s, t)=\frac{1}{n} \sum_{m=0}^{n-1} \sum_{k=-m}^{m} B_{k}(t) \exp \left(\frac{2 k s}{T}\right)
$$

satisfies that

$$
\sum_{p, q=1}^{k}\left\langle A_{p}^{*} B_{n}\left(t_{q}-t_{p}\right) A_{q} a, a\right\rangle_{\varepsilon} \geq 0
$$

for any $k \geq 1$. Since $B_{n}(s, t)$ converges to $B(s, t)$, it follows that $B(s, t)$ also verifies the above inequality, and, consequently is the covariance function of some periodically $\Gamma$-correlated process.

In the discrete periodically $\Gamma$-correlated case [12], similarly with the scalar case, was proved that any periodically $\Gamma$-correlated process $\left\{f_{n}\right\}_{n \in \mathbb{Z}}$ is $\Gamma$ harmonizable [11] i.e., there exists an $\mathcal{L}(\mathcal{E})$-valued semispectral measure (bimeasure) $K$ on $\mathbb{T}^{2}$ such that

$$
\Gamma\left[f_{m}, f_{n}\right]=\iint_{\mathbb{T}^{2}} \chi(t)^{m} \chi(s)^{-n} K(\mathrm{~d} t, \mathrm{~d} s),
$$


where $\chi(t)^{n}=\mathrm{e}^{-2 \pi i n t}$.

Moreover, the support of the $\mathcal{L}(\mathcal{E})$-valued semispectral bimeasure attached to a discrete periodically $\Gamma$-correlated process with the period $T \geq 1$ is concentrated on $2 T-1$ equidistant straight line segments $v=u-2 k \pi k / T$, $k \in\{0, \pm 1, \ldots \pm(T-1)\}$ parallel to the diagonal of the square $[0,2 \pi] \times[0,2 \pi]$. Obvious if $T=1$ then the $\Gamma$-harmonizable process $\left\{f_{n}\right\}_{n \in \mathbb{Z}}$ is stationary $\Gamma$ correlated and the support is concentrated only on the diagonal of the square. In the continuous parameter case, this nice property is no longer valid even in the scalar case (see [1]). Only on supplementary conditions, some particular periodically $\Gamma$-correlated processes with continuous time will become $\Gamma$-harmonizable, and similarly, the support of the bimeasure will be on parallel equidistant straight lines in the plane.

A process $\left\{f_{t}\right\}_{t \in \mathbb{R}}$ is strongly $\Gamma$-harmonizable if the correlation function $\Gamma_{f}(s, t)$ can be expressed as

$$
\Gamma_{f}(s, t)=\iint_{\mathbb{R}^{2}} \mathrm{e}^{i(s u-t v)} K(\mathrm{~d} u, \mathrm{~d} v) \quad(s, t \in \mathbb{R}),
$$

for some positive definite $\mathcal{L}(\mathcal{E})$-valued bimeasure $K$ of bounded variation.

A process $\left\{f_{t}\right\}_{t \in \mathbb{R}}$ is weakly $\Gamma$-harmonizable if its correlation function can be expressed in the form $(3.9)$ for some $\mathcal{L}(\mathcal{E})$-valued bimeasure $K$ of finite variation.

Similarly as in [3], in the supplementary condition of strongly harmonizability can be proved the following

Proposition 3.2. Let $\left\{f_{t}\right\}_{t \in \mathbb{R}}$ be a strongly $\Gamma$-harmonizable process in the complete correlated action $\{\mathcal{E}, \mathcal{H}, \Gamma\}$. Then $\left\{f_{t}\right\}_{t \in \mathbb{R}}$ is periodically $\Gamma$-correlated processes with period $T$ if and only if the support of $K$ is in the set $\Delta$, where

$$
\Delta=\left\{(u, v) \in \mathbb{R}^{2} ; v=u-2 \pi k / T, k \in \mathbb{Z}\right\} .
$$

Proof. For any $s, t \in \mathbb{R}$, since $\mathrm{e}^{i T(u-v)}=1$ for $(u, v) \in \Delta$ we have

$$
\begin{gathered}
\Gamma_{f}(s+T, t+T)=\iint_{\Delta} \mathrm{e}^{i[(s+T) u-(t+T) v]} K(\mathrm{~d} u, \mathrm{~d} v)= \\
=\iint_{\Delta} \mathrm{e}^{i(s u-t v)} \mathrm{e}^{i T(u-v)} K(\mathrm{~d} u, \mathrm{~d} v)=\iint_{\Delta} \mathrm{e}^{i(s u-t v)} K(\mathrm{~d} u, \mathrm{~d} v)= \\
=\iint_{\mathbb{R}^{2}} \mathrm{e}^{i(s u-t v)} K(\mathrm{~d} u, \mathrm{~d} v)=\Gamma_{f}(s, t),
\end{gathered}
$$

therefore $\left\{f_{t}\right\}_{t \in \mathbb{R}}$ is a periodically $\Gamma$-correlated process with period $T$. 
Conversely, if $\left\{f_{t}\right\}_{t \in \mathbb{R}}$ is periodically $\Gamma$-correlated with period $T$, then for any $N \geq 1$ and $s, t \in \mathbb{R}$ we have

$$
\begin{aligned}
& \Gamma_{f}(s, t)=\frac{1}{2 N+1} \sum_{k=-N}^{N} \Gamma_{f}(s+k T, t+k T)= \\
= & \frac{1}{2 N+1} \sum_{k=-N}^{N} \iint_{\mathbb{R}^{2}} \mathrm{e}^{i[(s+k T) u-(t+k T) v]} K(\mathrm{~d} u, \mathrm{~d} v)= \\
= & \iint_{\mathbb{R}^{2}} \frac{\sin \left[\left(N+\frac{1}{2}\right) T(u-v)\right]}{2\left(N+\frac{1}{2}\right) \sin \frac{T(u-v)}{2}} \mathrm{e}^{i(s u-t v)} K(\mathrm{~d} u, \mathrm{~d} v) .
\end{aligned}
$$

Since the fraction under the last integral has the property that take the value 1 on $\Delta$, is bounded and continuous on $\mathbb{R}$ and converges pointwise to 1 as $N \rightarrow \infty$, by the bounded convergence theorem we have that

$$
\Gamma_{f}(s, t)=\iint_{\Delta} \mathrm{e}^{i(s u-t v)} K(\mathrm{~d} u, \mathrm{~d} v)
$$

which implies that the support of $K$ is in the set $\Delta$.

The previous Proposition is valid in the weakly $\Gamma$-harmonizable case, too, and similarly [5] can be proved the following

Proposition 3.3. Let $\left\{f_{t}\right\}_{t \in \mathbb{R}}$ be a weakly $\Gamma$-harmonizable process in the complete correlated action $\{\mathcal{E}, \mathcal{H}, \Gamma\}$. Then $\left\{f_{t}\right\}_{t \in \mathbb{R}}$ is periodically $\Gamma$-correlated processes with period $T$ if and only if the support of $K$ is in the set $\Delta$ given by $(3.10)$.

It is known that in the discrete case, any periodically $\Gamma$-correlated process is $\Gamma$-harmonizable and, moreover, to each periodically $\Gamma$-correlated process we can attach a stationary $\Gamma$-correlated process, helping us in solving most of the prediction problems by a "stationarization procedure". In the continuous case, this fact can not be done so simply, but at least in the strongly $\Gamma$ harmonizable case a stationarization can be done as follows.

Proposition 3.4. Let $\left\{f_{t}\right\}_{t \in \mathbb{R}}$ be a strongly $\Gamma$-harmonizable process. If $\left\{f_{t}\right\}_{t \in \mathbb{R}}$ is also periodically $\Gamma$-correlated processes with period $T>0$, then it can be represented into the form

$$
V_{f_{t}}=\sum_{k \in \mathbb{Z}} \exp \left(\frac{2 \pi i k t}{T}\right) V_{g_{k}(t)} \quad(t \in \mathbb{R})
$$


where $\left\{g_{k}(t)\right\}_{k \in \mathbb{Z}}$ is a family of stationary $\Gamma$-cross-correlated processes given by

$$
V_{g_{k}(t)}=\int_{0}^{\frac{2 \pi}{T}} \mathrm{e}^{i t u} \xi\left(\mathrm{d} u+\frac{2 \pi}{T}\right) \quad(t \in \mathbb{R}),
$$

$\xi$ being an $\mathcal{L}(\mathcal{E}, \mathcal{K})$-valued measure on $\mathbb{R}$.

Proof. Let $\sigma_{k}=\left[\frac{2 \pi}{T}, \frac{2 \pi(k+1)}{T}\right)$ for $k \in \mathbb{Z}$. It follows that $\left\{\sigma_{k} ; k \in \mathbb{Z}\right\}$ is a countable partition of $\mathbb{R}$. The process $\left\{f_{t}\right\}_{t \in \mathbb{R}}$ being a strongly $\Gamma$-harmonizable one, $\Gamma_{f}(s, t)$ is given by $(3.9)$ with the $\mathcal{L}(\mathcal{E})$-valued bimeasure $K$ on $\mathbb{R}^{2}$. If we consider the corresponding Naimark spectral dilation $[\mathcal{K}, W, E]$ of $K$, then, up to a unitary equivalence, the $\mathcal{L}(\mathcal{E}, \mathcal{K})$ - valued measure $\xi(\sigma)=E(\sigma, \cdot) W$ is a representing measure of $\left\{f_{t}\right\}_{t \in \mathbb{R}}$, that is

$$
V_{f_{t}}=\int_{\mathbb{R}} \mathrm{e}^{i t u} \xi(\mathrm{d} u)
$$

For $k \in \mathbb{Z}$ let us consider the $\mathcal{L}(\mathcal{E}, \mathcal{K})$ - valued measures $\xi_{k}$ obtained by $\xi_{k}(\sigma)=\xi\left(\sigma \cap \sigma_{k}\right), \sigma \in \mathcal{B}(\mathbb{R})$, and the process $\left\{h_{k}(t)\right\}$ defined by

$$
V_{h_{k}(t)}=\int_{\sigma_{k}} \mathrm{e}^{i t u} \xi(\mathrm{d} u)=\int_{\mathbb{R}} \mathrm{e}^{i t u} \xi_{k}(\mathrm{~d} u) .
$$

It follows that $V_{f_{t}}=\sum_{k \in \mathbb{Z}} V_{h_{k}(t)}$ and

$V_{h_{k}(t)}=\int_{\frac{2 \pi k}{T}}^{\frac{2 \pi(k+1)}{T}} \mathrm{e}^{i t u} \xi(\mathrm{d} u)=\exp \left(\frac{2 \pi i k t}{T}\right) \int_{0}^{\frac{2 \pi}{T}} \mathrm{e}^{i t u} \xi\left(\mathrm{d} u+\frac{2 \pi k}{T}\right)=\exp \left(\frac{2 \pi i k t}{T}\right) V_{g_{k}(t)}$.

Therefore (3.11) is obtained. Since $\left\{f_{t}\right\}_{t \in \mathbb{R}}$ is a periodically $\Gamma$-correlated too, using the discrete case of Gladyshev's theorem in the complete correlated case $\{\mathcal{E}, \mathcal{H}, \Gamma\}[12]$ it follows that

$$
\Gamma_{j k}(s, t)=\Gamma\left[g_{j}(s), g_{k}(t)\right]=\int_{0}^{\frac{2 \pi}{T}} \mathrm{e}^{i(t-s) u} F_{k-j}\left(\mathrm{~d} u+\frac{2 \pi j}{T}\right),
$$

that is $\left\{g_{k}(t)\right\}_{k \in \mathbb{Z}}$ is a family of stationary $\Gamma$-cross-correlated processes attached to $\left\{f_{t}\right\}_{t \in \mathbb{R}}$ as above.

Taking into account the existence of the stationary dilation in this case, using the $\Gamma$-orthogonal projection on a right $\mathcal{L}(\mathcal{E})$-submodule given by the Proposition 1.1, the predictable part can be obtained.

Acknowledgement. This work is partially supported by UEFISCDI Grant PN-II-ID-PCE-2011-3-0119 


\section{References}

[1] E. G. Gladyshev, Periodically and almost-periodically correlated random processes with continuous time parameter, Theory of Probability and Appl., 8, (1963), 173-177.

[2] H. Helson and D. Lowdenslager, Prediction theory and Fourier series in several variables I and II, Acta Math., 105, (1961), 175-213.

[3] H. L. Hurd, Representation of strongly harmonizable periodically correlated processes and their covariance, J. Multivar. Anal., 29, (1989), 53-67.

[4] P. Masani, Recent trends in multivariate prediction theory, Math. Research 85, Acad. Press, New York, 1966

[5] D. K. Chang and M. M. Rao, Special representation of weakly harmonizable processes, Stochastic Anal. Appl., 6, (1988), 169-189.

[6] I. Suciu and I. Valusescu, Factorization of semispectral measures, Rev. Roumaine Math. Pures et Appl., 21 (6), (1976), 773-793.

[7] I. Suciu and I. Valusescu, Essential parameters in prediction, Rev. Roumaine Math. Pures et Appl., 22 (10), (1977), 1477-1495.

[8] I. Suciu and I. Valusescu, Factorization theorems and prediction theory, Rev. Roumaine Math. Pures et Appl., 23 (9), (1978), 1393-1423.

[9] I. Suciu and I. Valusescu, A linear filtering problem in complete correlated actions, Journal of Multivariate Analysis, 9 (4), (1979), 559-613.

[10] I. Valusescu, Continuous stationary processes in complete correlated actions, The P. Masani Volume (V. Mandrekar and H. Salehi eds.), North-Holland Pub. Comp., 1983

[11] I. Valusescu, Operatorial non-stationary harmonizable processes, Z. Angew. Math. Mech., 76 (suppl. 2), (1996), 695-696.

[12] I. Valusescu, A linear filter for the operatorial prediction of a periodically correlated process, Rev. Roum. Math. Pures et Appl., 54 (1), (2009), 53-67.

Ilie Valuşescu

Romanian Academy

Institute of Mathematics "Simion Stoilow"

P.O.Box 1-764, 014700 Bucharest

Romania

E-mail: Ilie.Valusescu@imar.ro

Received: 01.02.2012

Accepted: 21.06.2012 